\title{
cMET inhibitor crizotinib impairs angiogenesis and reduces tumor burden in the C3(1)-Tag model of basal-like breast cancer
}

\author{
Alyssa J. Cozzo ${ }^{1 \dagger}$, Sneha Sundaram ${ }^{1 \dagger}$, Ottavia Zattra ${ }^{1}$, Yuanyuan Qin ${ }^{1}$, Alex J. Freemerman ${ }^{1}$, Luma Essaid $^{1}$, \\ David B. Darr ${ }^{3}$, Stephanie A. Montgomery ${ }^{3,6}$, Kirk K. McNaughton², J. Ashley Ezzell2, Joseph A. Galanko4, \\ Melissa A. Troester ${ }^{3,5}$ and Liza Makowski ${ }^{1,3,4^{*}}$
}

\begin{abstract}
Epidemiologic studies have associated obesity with increased risk of the aggressive basal-like breast cancer (BBC) subtype. Hepatocyte growth factor (HGF) signaling through its receptor, CMET, is elevated in obesity and is a pro-tumorigenic pathway strongly associated with BBC. We previously reported that high fat diet (HFD) elevated HGF, CMET, and phospho-cMET in normal mammary gland, with accelerated tumor development, compared to low fat diet (LFD)-fed lean controls in a murine model of BBC. We also showed that weight loss resulted in a significant reversal of HFDinduced effects on latency and elevation of HGF/CMET signaling in normal mammary and CMET in normal mammary and tumors. Here, we sought to inhibit BBC tumor progression in LFD- and HFD-fed C3(1)-Tag BBC mice using a small molecule cMET inhibitor, and began crizotinib treatment $(50 \mathrm{mg} / \mathrm{kg}$ body weight by oral gavage) upon identification of the first palpable tumor. We next investigated if administering crizotinib in a window prior to tumor development would inhibit or delay BBC tumorigenesis. Treatment: Crizotinib significantly reduced mean tumor burden by 27.96 and $37.29 \%$, and mean tumor vascularity by 35.04 and $33.52 \%$, in our LFD- and HFD-fed C3(1)-Tag BBC mice, respectively. Prevention: Crizotinib significantly accelerated primary tumor progression in both diet groups but had no effect on total tumor progression or total tumor burden. In sum, cMET inhibition by crizotinib limited tumor development and microvascular density in basal-like tumor-bearing mice but did not appear to be an effective preventive agent for BBC.
\end{abstract}

Keywords: Hepatocyte growth factor, Triple negative breast cancer, Obesity, Microenvironment, Microvascular density

\section{Background}

Basal-like breast cancer (BBC) accounts for $15-20 \%$ of total breast cancers, with a higher prevalence in young and minority women such as African Americans and Hispanics (Carey et al. 2006; Boyle 2012). BBC is typically estrogen receptor (ER), progesterone receptor (PR) and human epidermal growth factor receptor-2 (HER2) negative (so called "triple-negative") and is highly aggressive,

\footnotetext{
*Correspondence: liza.makowski@unc.edu

${ }^{\dagger}$ Alyssa J. Cozzo and Sneha Sundaram contributed equally to this work ${ }^{1}$ Department of Nutrition, University of North Carolina at Chapel Hill, Chapel Hill, NC 27599, USA

Full list of author information is available at the end of the article
}

exhibiting an early pattern of metastasis and poor overall prognosis. Thus, BBC presents a formidable challenge, as it lacks the molecular targets for current targeted drug treatments. High body mass index (BMI) is associated with poorer prognosis in breast cancer patients, including increased risk of lymph node metastasis, vascular invasion, disease recurrence, and mortality (Dawood et al. 2008; Calle et al. 2003; Biglia et al. 2013). Epidemiologic studies indicate that obesity is strongly associated with the BBC subtype in both pre- and post-menopausal women (Biglia et al. 2013; Boyle 2012). In obesity, rapid expansion in mammary adipose tissue leads to alterations in the stroma that mediate normal and tumor

\section{黛 Springer}


microenvironment dysfunction yet are poorly understood in breast cancer risk and progression (Iyengar et al. 2015; Ford et al. 2013; Johnson et al. 2012; Sundaram et al. 2013b). Given that obesity has increasing prevalence and is one of few modifiable risk factors for breast cancer, it is important to better elucidate the mechanisms for this obesity-associated cancer.

We have shown that $B B C$ is characterized by unique epithelial-stromal interactions, which likely play a role in BBC etiology (Camp et al. 2011; Casbas-Hernandez et al. 2013; Brauer et al. 2013; Stewart et al. 2012). An elevated level of hepatocyte growth factor (HGF), a pleiotropic growth factor that signals through the receptor tyrosine kinase cMET, is characteristic of BBC. Elevation of HGF is also seen in obese patients (Bell et al. 2006). HGF/ cMET signaling initiates an invasive growth program that promotes cell migration, invasion, proliferation, and angiogenesis (Mizuno and Nakamura 2013). Endothelial cell upregulation of cMET has been attributed to inherent or acquired resistance to antiangiogenic therapies targeting vascular endothelial growth factor (VEGF) in patients (Shojaei et al. 2010; Ding et al. 2003). Marking the first work in preclinical models paralleling human epidemiologic BBC findings, we used C3(1)-TAg mice, a unique genetically engineered mouse model (GEMM) of spontaneous $\mathrm{BBC}$, to demonstrate that high fat diet (HFD)-induced obesity accelerated onset of tumor development and increased tumor aggressiveness as compared to low fat diet (LFD)-fed lean controls (Sundaram et al. 2013a). HFD also increased mammary gland HGF concentration and enhanced expression and activation of cMET. Using primary murine fibroblasts isolated from mammary glands or tumors, we further reported that obesity increased HGF production by mammary gland normal- and cancer-associated fibroblasts (NAF and CAF) (Sundaram et al. 2013a). Through signaling inhibition via an HGF blocking antibody, we showed that obese CAF-induced epithelial cell migration occurred in an HGF-dependent mechanism. Furthermore, using the intervention strategy of weight loss prior to tumor latency, we reported that weight loss blunted effects of HFD-induced obesity on multiple tumor parameters compared to mice maintained on HFD. Importantly, HFD-induced elevation of HGF/cMET signaling in normal mammary gland and cMET in tumors was significantly reversed with weight loss in C3(1)-Tag mice, with a concomitant and complete reversal of HFD-driven tumor progression (Sundaram et al. 2014b).

Given the precedent for the role of HGF signaling in invasive breast cancer (Gastaldi et al. 2010), a better understanding of HGF's role in BBC tumorigenesis was necessary. We hypothesized that inhibition of cMET signaling through crizotinib therapy (PF-02341066) would reduce HFD-induced $B B C$. We first sought to inhibit tumor progression in existing tumors and began crizotinib treatment upon identification of the first palpable tumor. Crizotinib significantly reduced total tumor burden in both LFD- and HFD-fed C3(1)-Tag mice, with a corresponding reduction in microvascular density. We next investigated whether we could inhibit or delay tumorigenesis by treating C3(1)-Tag mice with crizotinib prior to tumor development. Crizotinib treatment paradoxically increased progression of the initially detected tumor in both diet groups. However, at sacrifice there were no differences between diet or treatment groups in total preneoplastic lesions, total tumor progression or tumor burden. In summary, cMET inhibition disrupted tumor vascularization and limited subsequent BBC tumor development in tumor-bearing mice. Our results suggest that reduction of microvascular density through cMET inhibition may be a viable therapeutic target in the treatment of BBC.

\section{Results}

Basal-like tumor latency was accelerated by HFD

Adult female C3(1)-Tag mice were randomly assigned to diet groups at 10 weeks of age (Additional file 1: Figure S1A, Model of Treatment study design) and body weight and body composition were monitored. Tumor latency and progression were tracked by palpation and calipers. Body weight was not significantly altered by diet or vehicle or drug treatment group for the study duration (Additional file 1: Figure S1B). Consistent with our previously reported results (Sundaram et al. 2013a), C3(1)-Tag mice fed HFD exhibited significantly decreased tumor latency compared with mice fed LFD (LFD median 17.3 weeks,; HFD median 15.5 weeks; $\mathrm{P}<0.0001$, Fig. 1a). Using a Chi square test with a degree of freedom of 1 , LFD vs HFD equaled 15.72. Adiposity as measured by MRI was increased with HFD but did not reach significance (Fig. 1b). Oral gavage of vehicle or drug slightly reduced adiposity among all groups, irrespective of diet or treatment (Fig. 1b). Gonadal fat pad mass was significantly greater in mice fed HFD compared to mice fed LFD $(P=0.0173$, Fig. 1c).

\section{Crizotinib treatment inhibited secondary tumor development}

Crizotinib treatment significantly reduced total tumor burden, by 27.96 and $37.29 \%$ in LFD- and HFD-fed C3(1)Tag mice, respectively, compared to mice treated with vehicle $(P=0.0085$, Fig. $1 d)$. A minor diet effect on tumor burden was detected; HFD-fed animals in both vehicleand crizotinib-treated groups showed reduced tumor burden at sacrifice compared to LFD-fed mice ( $P=0.0491$, Fig. 1d). Tumor progression of all palpable tumors was 


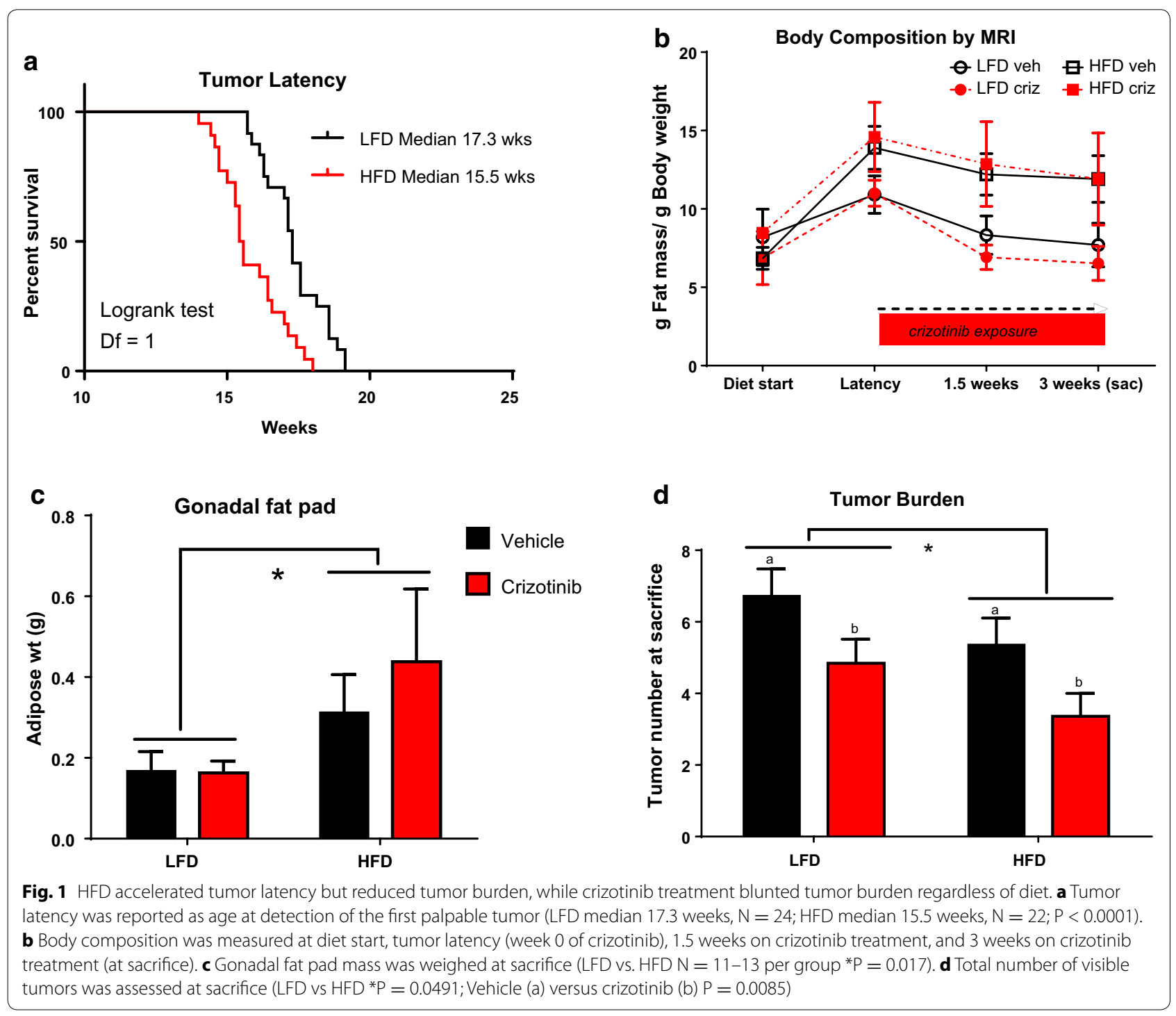

monitored using calipers throughout the 3-week measurement period between latency and sacrifice. No differences by diet or treatment group in primary tumor progression were detected (Additional file 1: Figure S1C).

\section{Crizotinib disrupted tumor vascularization}

CD31, a marker of angiogenic and lymphangiogenic microvessel density (MVD) (Choi et al. 2005), was measured by IHC (Fig. 2a-e). Crizotinib administration significantly reduced mean tumor MVD by 35.04 and $33.52 \%$ in LFD and HFD groups, respectively $(\mathrm{P}=0.014$, Fig. $2 \mathrm{f})$. There were no diet effects on CD31 positivity. Total cMET staining did not differ by diet or treatment group (Additional file 2: Figure S2A), while phosphorylated (active) cMET was significantly higher in mice fed HFD $(\mathrm{P}=0.014$, Additional file 2: Figure $\mathrm{S} 2 \mathrm{~B})$.

\section{Crizotinib prophylactic treatment did not affect body weight or adiposity}

As crizotinib treatment reduced tumor burden in tumorbearing mice, we next investigated whether we could inhibit or delay tumorigenesis by treating with crizotinib prior to tumor development. C3(1)-Tag tumors progress along the following timeline: atypical hyperplasia $(\mathrm{AH})$ of the mammary ductal epithelium at 8 weeks of age, mammary intraepithelial neoplasia (resembling human carcinoma in situ [CIS]) at 12 weeks of age, and invasive carcinomas at 16 weeks of age with $100 \%$ penetrance (Green et al. 2000). Thus, in the prevention arm of the study, mice were started on diet 2 weeks earlier than in the treatment study above to ensure crizotinib administration occurred within the primary window of AH/CIS precursor lesions. Starting at 8 weeks of age, mice were randomly assigned to LFD 


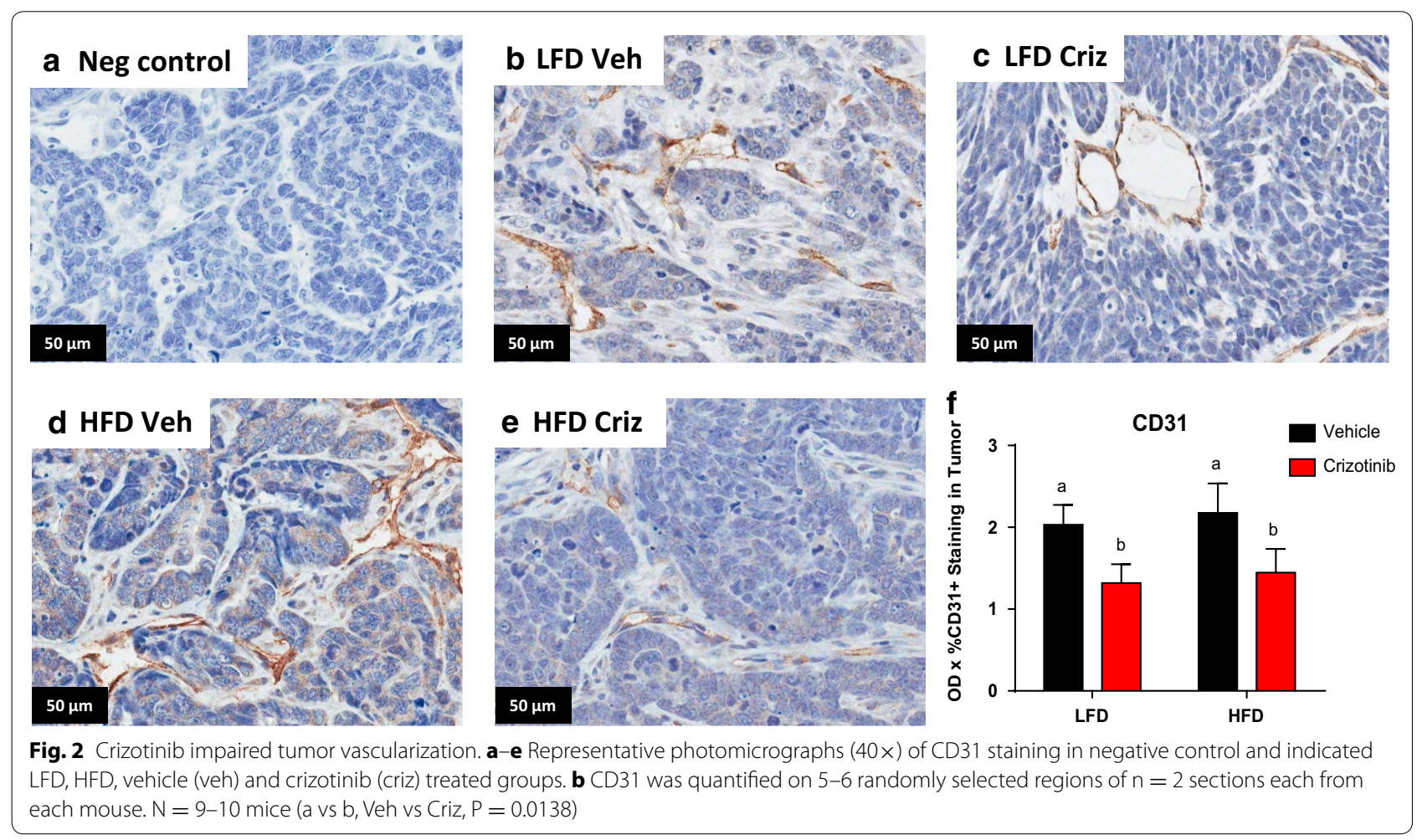

or HFD, with crizotinib treatment beginning at 9 weeks of age and continuing until 12 weeks of age (Additional file 3: Figure S3A). Mice fed HFD diet gained significantly more weight than the LFD mice, with greater body weights from 9 to 16 weeks of age ( $P<0.05$, Additional file 3: Figure S3B). Body composition differed significantly between LFD- and HFD-fed mice beginning at 1 week on diet (9 weeks of age) and remained significant until sacrifice $(\mathrm{P}<0.0001$ for all data points, Fig. 3a). Crizotinib- and vehicle-treated mice fed HFD had significantly greater gonadal fat pad mass when compared to LFD-fed mice $(\mathrm{P}<0.0001$, Fig. 3b). No difference was detected in body weight or adiposity between the crizotinib and vehicle treated mice (Fig. 3a, b).

\section{Crizotinib prophylactic treatment increased tumor} progression but did not significantly alter tumor burden or precursor lesions

Upon initiation of crizotinib or vehicle administration mice were palpated twice weekly for detection of tumor onset. Median tumor latency did not vary significantly between diet or treatment groups (LFD vehicle-treated median 15.0 weeks; LFD crizotinib-treated median 16.1 weeks; HFD vehicle median 16.0 weeks; HFD crizotinib median 16.2 weeks, Fig. 3c). Progression of the primary, initially detected tumor was significantly increased with crizotinib treatment in both diet groups $(\mathrm{P}=0.04$, Additional file 3 : Figure S3C). However, when all tumors were considered there were no differences between diet or treatment groups in total tumor progression (Additional file 3: Figure S3D) or tumor burden (Fig. 3d) at sacrifice. Non-tumor mammary tissue was analyzed for $\mathrm{AH}$ and CIS premalignant lesions in of HFD-fed vehicle- or crizotinib-treated mice. There were no significant diet- or crizotinib-mediated effects on precursor lesion formation detected (Additional file 3: Figure S3E).

\section{Discussion}

The HGF/cMET pathway is regulated by body weight and is relevant to $\mathrm{BBC}$. HGF is the only known ligand for cMET, and the HGF/cMET signaling pathway has long been studied in normal development, ductal morphogenesis (Garner et al. 2011), invasive breast cancer (Tuck et al. 1996; Yamashita et al. 1994; Elliott et al. 2002; Wang et al. 1994; Jin et al. 1997; Beviglia et al. 1997), and invasive biology of several other cancers due to its angiogenic, mitogenic, and morphogenic effects (Stella et al. 2005; Sam et al. 2007). Notably, single nucleotide polymorphisms in the $M E T$ gene may be associated with metastatic breast cancer (Liu 2015). In patients, we demonstrated that $86 \%$ of BBC expressed an HGF/cMET activation signature through gene expression (Casbas-Hernandez et al. 2013). Furthermore, a recent comprehensive meta-analysis including over 6000 cases showed that cMET overexpression is significantly associated with poor survival in breast cancer patients, particularly among patients 

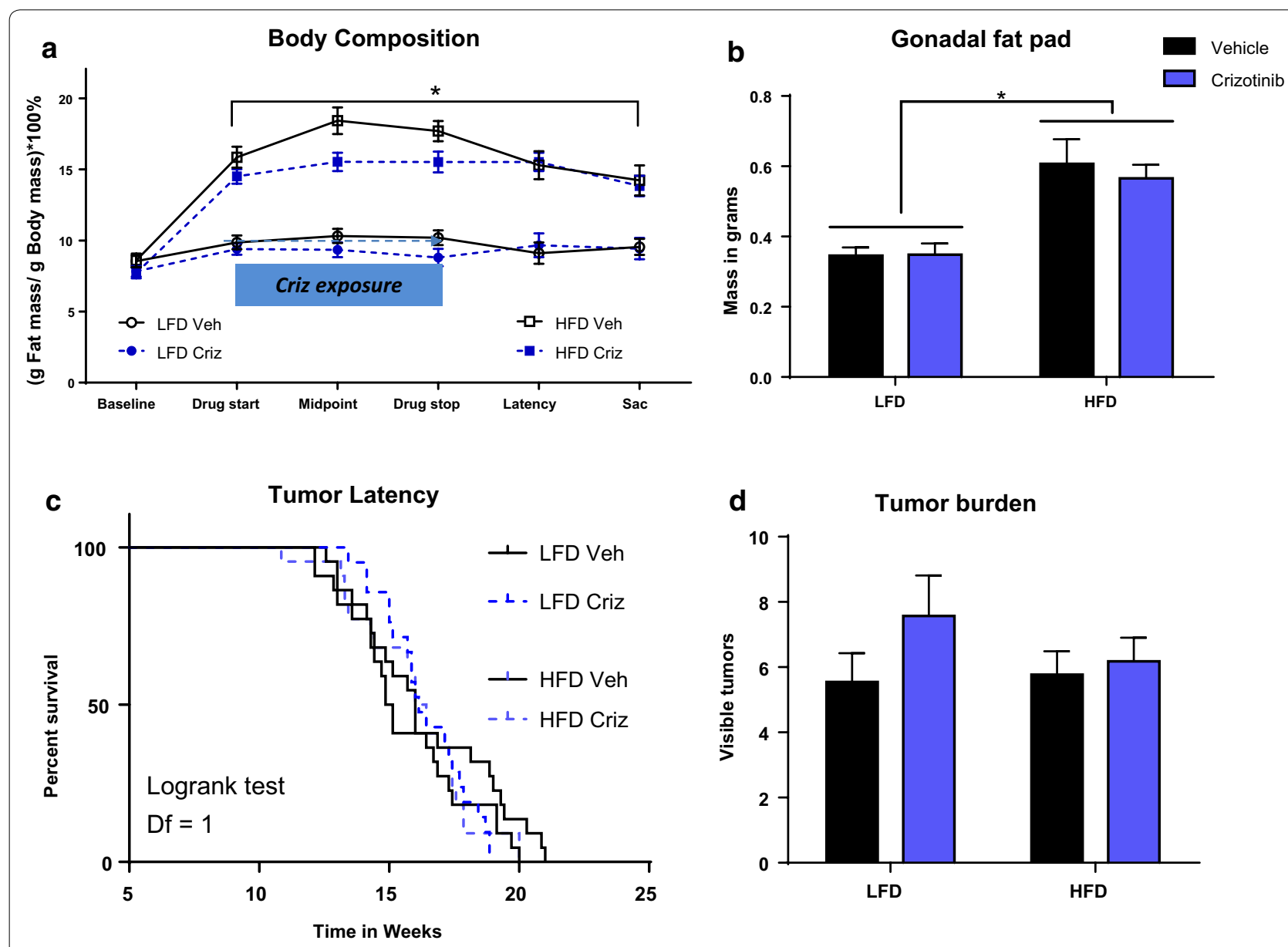

Fig. 3 Preventive administration of crizotinib prior to tumor onset did not alter tumor parameters. Diet was started at 8 weeks of age (baseline). a Body composition was measured by MRI (LFD vs. HFD *P < 0.0001). b Gonadal fat pad mass was determined at sacrifice (LFD vs. HFD *P < 0.0001$)$. c Upon initiation of crizotinib treatment ( $50 \mathrm{mg} / \mathrm{kg}$ by oral gavage) at 9 weeks of age, mice were palpated twice weekly for tumor onset. Median tumor latency did not differ with diet or treatment (LFD veh-treated median 15.0 weeks, $\mathrm{N}=22$; LFD criz-treated median 16.1 weeks, $\mathrm{N}=21$; HFD veh-treated median 16.0 weeks; HFD criz-treated median 16.2 weeks). d Total tumor burden was assessed at sacrifice

with triple-negative breast cancer (Yan et al. 2015). Obesity mediates, and can exacerbate, both normal and tumor microenvironment dysfunction (Johnson et al. 2012; Sundaram et al. 2013b). HGF is an excellent candidate mediator of obesity-induced effects on cancer, as serum HGF is elevated in obese individuals and reduced with weight loss (Hiratsuka et al. 2005; Bell et al. 2006; Swierczynski et al. 2005). Moreover, adipose-derived HGF has been detected in normal and malignant breast tissue (Tuck et al. 1996).

We have previously demonstrated that HFD exposure during adulthood increased cMET expression and activation in normal mammary and tumors (Sundaram et al. 2013a). Diet-induced upregulation of cMET in tumors was also evident in mice fed HFD from weaning and could be reversed by weight loss (Sundaram et al. 2014b). Our previous work further revealed that HFDinduced weight gain resulted in increased mammary gland HGF that was reversed with weight loss (Sundaram et al. 2014b). Reduction in HGF due to weight loss correlated with diminished tumor progression or deceleration of tumor latency, depending on whether the diet was initiated at weaning or adult onset, respectively. Altogether, our previously published studies on HGF/cMET implicated HGF/cMET signaling as a mediator of obesity-driven BBC tumor aggression. Therefore, this study aimed to specifically inhibit cMET signaling as a potential $\mathrm{BBC}$ treatment or prevention strategy, hypothesizing that disruption of HGF/cMET signaling would mimic the effects of weight loss on $\mathrm{BBC}$ tumorigenesis.

C3(1)Tag mice are a GEMM that develop BBC in $100 \%$ of female mice (Green et al. 2000). Initial characterization of the C3(1)-Tag model reported grossly palpable tumors at $~ 16$ weeks of age; in our hands, median tumor latency occurs between 15 and 19 weeks, dependent on diet 
composition and age at diet start (Sundaram et al. 2013b, 2014a, b). Several groups have reported "windows of susceptibility" during which HFD and/or obesity may play a disproportionately greater role in promoting breast cancer onset (Biro and Deardorff 2013; Sundaram et al. 2013b). As shown here, in the crizotinib treatment arm of our study, initiating diets at 10 weeks of age resulted in detection of tumor latency two weeks earlier in mice that were fed HFD compared to mice fed LFD, consistent with our previous C(3)1-Tag studies in which identical diets were initiated at the same age (Sundaram et al. 2013a). However, in the crizotinib prevention arm of our study, in which HFD was initiated at 8 weeks of age, we did not see this diet effect of accelerated latency-these results parallel our second previously published study, in which female C3(1)Tag mice were weaned onto LFD or HFD and no difference in latency was observed (Sundaram et al. 2014b). Interestingly, pubertal exposure to HFD has been linked to stunted mammary duct elongation and reduced mammary epithelial cell proliferation in murine models (Olson et al. 2010), a finding that was not seen in mice started on diet at 10 weeks of age or older. Collectively, our results here and in our previous studies support the concept that exposures extrinsic to the cancer cell (i.e., diet-induced alterations in the mammary microenvironment) can impact tumorigenesis, while age at diet start may be an important variable contributing to diet effects on tumor latency.

Herein, we showed that crizotinib treatment after BBC latency inhibited subsequent tumor formation such that total tumor burden was reduced at sacrifice, regardless of diet. The degree of tumor inhibition was paralleled by a similar degree of suppression of MVD, also irrespective of diet. Reduction in MVD in our crizotinib-treated tumor-bearing C3(1)-Tag mice could explain the significant reduction of tumor burden compared to vehicle-treated controls. In tumors, an "angiogenic switch" occurs, in which an increase in MVD in and near the tumor allows for tumor survival and metastasis (Lin and Pollard 2007). Increases in HGF in the tumor microenvironment contribute to this angiogenic switch (Wagatsuma et al. 1998; Abounader and Laterra 2005), while cMET signaling by the cancer cells facilitates invasion and migration away from the hypoxic interior of the tumor, entry into the new and leaky vessels, and metastasis to distant locations (Gastaldi et al. 2010). Indeed, obesity-promoted HGF production by fibroblasts, adipocytes, macrophages, and endothelial cells (Sundaram et al. 2013a, 2014b; Casbas-Hernandez et al. 2011, 2013; Mizuno and Nakamura 2013) may be a unique mechanism to increase blood vessel density and alleviate the hypoxia of obese adipose tissue. The fact that no changes were detected in latency in the prevention arm of our study suggests that early changes in the HGF/cMET axis (i.e., prior to invasive carcinoma) may not be as easily targeted, or are not causative in BBC tumorigenesis.

Initial mechanism-of-action studies for crizotinib showed that dose-dependent inhibition of cMET in gastric carcinoma, glioblastoma, and prostate carcinoma resulted in reduction of MVD as measured by CD31 (Zou et al. 2007). Inhibition of cMET as an effective anti-angiogenic agent has also been shown in xenograft models of aggressive cancers such as lung (Puri et al. 2007) and pancreatic cancers (You et al. 2011). Moreover, the use of other cMET inhibitors in triple-negative breast cancer models such as ours has yielded promising results in preclinical studies (Sameni et al. 2015). However, to date clinical trials using the cMET inhibitors tivantinib or onartuzamab in isolation or in combination with chemotherapy have demonstrated little therapeutic benefit in metastatic breast cancer (Tolaney et al. 2015; Dieras et al. 2015; Sharma and Adjei 2011). Clinical trials investigating crizotinib alone [ClinicalTrials.gov:NCT 02101385 (Schneider 2016)] or in combination with antiVEGF therapy [ClinicalTrials.gov:NCT 02074878 (Baylor Breast Care Center 2015)] for the treatment of advanced triple negative breast cancer are currently underway. Breast cancer ranks as the fifth cause of death from cancer overall and is now the second cause of cancer death following lung cancer (Brewster et al. 2014; WHO 2014). With the growing global prevalence of obesity and the notable racial and ethnic disparities in BBC outcomes (Brewster et al. 2014), it is imperative that approaches are identified to effectively address the increased risk of breast cancer onset and progression to malignancy for an increasingly overweight and obese US population.

\section{Methods}

\section{Antibodies and drugs}

Crizotinib (PF-2341066 [(R)-3-[1-(2,6-dichloro-3-fluorophenyl)-ethoxy]-5-(1-piperidin-4-yl-1H-pyrazol-4-yl)pyridin-2-ylamine) was purchased from Selleck Chemical (Catalog No. S1068). Primary antibodies include: Rabbit anti-mouse CD31 (Abcam \#ab28364, Lot GR2123645; 1:400); Rabbit anti-mouse phospho-cMET (Abcam \#ab5662, Lot GR159296-1; 1:4000); Goat anti-mouse cMET (R\&D Systems \#AF527, Lot CTB0310091; 1:1000) diluted in Renoir Red Diluent (BM \#PD904H). Additional reagents included biotin-conjugated Goat anti-rabbit IgG (Jackson \#111-065-144, Lot 110335; 1:500); Donkey antigoat IgG (Jackson \#705-065-147, Lot 110544; 1:1000). ABC Elite (Vector \#PK-6100, 1:50) and 3,3' Diaminobenzidine (DAB) (Thermo Scientific \#TA-125-QHDX).

\section{Animals and diet}

Animal studies were performed with approval and in accordance with the guidelines of the Institutional Animal Care and Use Committee at the University of North 
Carolina at Chapel Hill. Animals were cared for according to the recommendations of the Panel on Euthanasia of the American Veterinary Medical Association. The veterinary care provided at UNC is in compliance with the Public Health Service Policy on Humane Care and Use of Laboratory Animals and meets the National Institutes of Health standards as set forth in the Guide for the Care and Use of Laboratory Animals (DHHS Publication No. (NIH) 85-23 Revised 1985). The animal facility is Association for Assessment and Accreditation of Laboratory Animal Care (AAALAC) approved and is responsible for the health and husbandry of animals. UNC also accepts as mandatory the PHS Policy on Humane Care and Use of Laboratory Animals be Awardee Institutions and NIH Principles for the Utilization and Care of Vertebrate Animals Used in Testing, Research, and Training. Animal studies comply with the ARRIVE guidelines. Mice were housed in a climate controlled Department of Laboratory Animal Medicine facility with a $12 \mathrm{~h}$ light:dark cycle and ad libitium access to food and water or special diets as defined below. Female C3(1)Tag mice were obtained in collaboration with the UNC Lineberger Comprehensive Cancer Center (LCCC) Mouse Phase I Unit (MP1U). C3(1)-Tag mice (Green et al. 2000), a model of $\mathrm{BBC}$, were generated by crossing heterozygous male mice with $\mathrm{FVB} / \mathrm{N}$ non-transgenic female mice.

For the tumor treatment study, $\mathrm{N}=46$ female C3(1)Tag mice were bred and maintained on chow diet (Harlan 2918) until nulliparous female were randomly assigned to LFD $(\mathrm{N}=24)$ and HFD $(\mathrm{N}=22)$ at 10 weeks of age. Diets obtained from Research Diets Inc. (New Brunswick, NJ, USA) were matched for protein, vitamins, and minerals, and provided $10 \% \mathrm{kcal}$ ("LFD"; \# D11012202); and $60 \% \mathrm{kcal}$ ("HFD"; \# D11012204) derived from fat. Diets were sucrosefree, and soy-free. Additional details of diet components are provided in Sundaram et al. (Sundaram et al. 2013a). Model of treatment study design is supplied in Additional file 1: Figure S1A). For the prevention study, during breeding and after weaning mice were put on Prolab Isopro RMH 3000 from LabDiet (St. Louis, MO, USA). At 8 weeks of age, nulliparous female C3(1)-Tag mice were randomly assigned to LFD $(\mathrm{N}=43)$ and HFD $(\mathrm{N}=45)$ diet groups (Model of treatment study design, Additional file 3: Figure S3A).

\section{Tumor latency, number, and progression}

Mice were monitored for tumor development by palpating twice weekly. Tumor latency defined as age in weeks at detection of first tumor. Tumor volumes were measured twice weekly over 3 weeks using calipers to measure the width (short diameter) and length (long diameter) in millimeters for each tumor. Tumor volumes were calculated using the formula: length $\times$ width $^{2} \times 0.5$. Tumor progression is reported as percent change in volume from latency to sacrifice 3 weeks later. Primary tumor progression refers to the first tumor identified; total tumor progression includes all tumors palpated. The total number of visible tumors per mouse was counted at sacrifice for total tumor burden.

\section{Crizotinib treatment}

Crizotinib dosage was $50 \mathrm{mg}$ crizotinib/kg of body weight (Zou et al. 2007). In the treatment study, crizotinib administration by oral gavage began at identification of the first palpable tumor and persisted for 3 weeks until sacrifice ( 5 days on drug, 2 days rest) (Additional file 1: Figure S1A). Briefly, drug was prepared by dissolving $20 \mathrm{mg}$ of crizotinib powder in $200 \mu \mathrm{L} 1 \mathrm{~N}$ hydrochloric acid $(\mathrm{HCl})$, then brought to a total volume of $1 \mathrm{~mL}$ with vehicle $(0.5 \%$ glucose in phosphate-buffered saline) to yield a $2 \times$ crizotinib solution. Immediately prior to gavage administration, the $2 x$ solution was diluted with an equal volume of vehicle to yield a $1 \mathrm{x}$ solution. In the prevention study, drug was prepared as a $1 \mathrm{x}$ solution by dissolving $10 \mathrm{mg}$ of crizotinib powder in $200 \mu \mathrm{L} 1 \mathrm{~N}$ hydrochloric acid $(\mathrm{HCl})$, then brought to a total volume of $1 \mathrm{~mL}$ with vehicle; crizotinib treatment began for all mice at 9 weeks of age and continued for 3 weeks ( 5 days on drug, 2 days rest).

\section{Body weight and composition}

Body weight was measured at start of diet and weekly until sacrifice. Body composition including lean mass, fat mass, free water content and total water content of nonanesthetized mice was measured using EchoMRI-100 quantitative magnetic resonance whole body composition analyzer (Echo Medical Systems, Houston, TX). Fat mass is presented as percent fat mass over total body weight (Sundaram et al. 2013a, 2014b). There were no significant changes in absolute lean mass in grams (data not shown).

\section{Tissue and blood collection}

Three weeks after detection of the first palpable tumor, mice were fasted for $6 \mathrm{~h}$ and anesthetized by an intraperitoneal (i.p.) injection of avertin (tribromoethanol/ amylene hydrate, $1.25 \%$ ) (Sigma Aldrich, St. Louis, MO). Blood was collected via cardiac puncture using an EDTAcoated syringe into $5 \mu \mathrm{L}$ of $250 \mathrm{mM}$ EDTA. Plasma was separated from other blood components by centrifugation at $10,000 \times g$ for $2 \mathrm{~min}$ at $4{ }^{\circ} \mathrm{C}$. Mammary tumors, unaffected inguinal mammary gland, liver, spleen, and lungs were flash frozen in liquid nitrogen or were placed into a cassette and formalin-fixed for immunohistochemistry (IHC) and H\&E analysis. All frozen samples were stored at $-80^{\circ} \mathrm{C}$ until analyzed.

\section{Immunohistochemistry}

Briefly, formalin-fixed and paraffin-embedded tissues were sectioned at 5 microns and mounted for histological staining 
(Sundaram et al. 2013a). Tissues were baked, deparaffinized, and hydrated. Following heat-induced epitope retrieval (Rodent Decloaker BM\#RD913L), slides were treated with $3 \%$ hydrogen peroxide in de-ionized water. Tissues were treated with Avidin/Biotin Block (Vector \#SP-2001) and exposed to primary antibodies (anti-CC3, anti-Ki67 antiCD31; anti-phospho-cMET; anti-cMET) diluted in Renoir Red Diluent at $4{ }^{\circ} \mathrm{C}$ overnight. Following incubation with biotin-conjugated secondary antibodies [Goat anti-rabbit IgG; Donkey anti-goat IgG] tissue sections were treated with $\mathrm{ABC}$ Elite and DAB. Digital immunohistochemistry quantification was performed following the protocol previously described in Sundaram et al. (2013a). Stained slides were scanned into the Aperio Scanscope CS system (Aperio Technologies, Vista, CA, USA) at a magnification of $40 \times$ and were quantified using the Aperio Imagescope software. Scanned slides were analyzed using algorithms as described previously (Sundaram et al. 2014a). N = 5-6 random areas from sections $(\mathrm{n}=2$ sections per mouse) were quantified and averaged per tumor per animal $(\mathrm{n}=9-10$ mice per diet or exposure group). Images shown are representative.

\section{Statistical analysis}

Data are expressed as mean \pm standard error of the mean (SEM). All means were compared by 2 way analysis of variance (ANOVA) with Tukey's post hoc test for statistical differences using GraphPad Prism 5 software (GraphPad Software, Inc. La Jolla, CA). Kaplan-Meier analyses were conducted using GraphPad Prism 5 software to estimate tumor latency. Log rank and Chi square tests were used to investigate differences among groups. P values $<0.05$ were considered statistically significant.

\section{Additional files}

Additional file 1: Figure S1. A) Model of Treatment Study design. At 10 weeks of age female mice were randomized to LFD or HFD and palpation began for identification of tumor onset. Vehicle or crizotinib treatment by oral gavage began at detection of first palpable tumor and lasted for 3 weeks, 5 days on and 2 days off. Mice were sacrificed at 3 weeks past tumor onset. Metabolic and other parameters were measured as indicated. B) Body weights did not differ by diet or treatment group. No significant differences were observed in C) primary or D) total tumor progression.

Additional file 2: Figure S2. A) Total MET staining did not differ by diet or treatment group. B) Phosphorylated (active) MET was significantly higher in mice fed HFD (2-way ANOVA $* P=0.0141)$.

Additional file 3: Figure S3. A) Model of Prevention Study design. At 8 weeks of age female mice were randomized to LFD or HFD. At 9 weeks of age vehicle or crizotinib treatment by oral gavage began and lasted for 3 weeks, 5 days on and 2 days off. Palpation began for identification of tumor onset also began at 9 weeks of age. Mice were sacrificed at 3 weeks past tumor onset. Metabolic and other parameters were measured as indicated. B) Mice fed HFD diet gained significantly more weight than the LFD mice, with greater body weights from 9 to 16 weeks of age (LFD vs HFD $\left.{ }^{*} P<0.05\right)$. Progression of the primary tumor was significantly increased with crizotinib treatment in both diet groups (a vs $b, P=0.036$ ), but had no effect on total tumor progression or total tumor burden.

\section{Authors' contributions}

SS and AJC carried out the mouse studies and assays. YQ, DBD, and AJF assisted with mouse studies. JG performed the statistical analysis. OZ, LE, KKM and JAE assisted with immunohistochemical analysis. MAT participated in its design. SAM is a veterinary pathologist who reviewed slides for pre-neoplastic lesions. SS, AJC and LM conceived of the study, and participated in its design, and helped to draft the manuscript. All authors read and approved the final manuscript.

\section{Author details}

${ }^{1}$ Department of Nutrition, University of North Carolina at Chapel Hill, Chapel Hill, NC 27599, USA. ${ }^{2}$ Department of Cell Biology and Physiology, University of North Carolina at Chapel Hill, Chapel Hill, NC 27599, USA. ${ }^{3}$ Lineberger Comprehensive Cancer Center, University of North Carolina at Chapel Hill, Chapel Hill, NC 27599, USA. ${ }^{4}$ Nutrition Obesity Research Center, University of North Carolina at Chapel Hill, Chapel Hill, NC 27599, USA. ${ }^{5}$ Department of Epidemiology, University of North Carolina at Chapel Hill, Chapel Hill, NC 27599, USA. ${ }^{6}$ Department of Pathology and Laboratory Medicine, University of North Carolina at Chapel Hill, Chapel Hill, NC 27599, USA.

\section{Acknowledgements}

UNC Mouse Phase 1 Phenotyping Core for excellent support. We acknowledge Liyang Zhao for her technical assistance.

\section{Competing interests}

The authors declare that they have no competing interests.

\section{Funding sources}

LM: NIH/NCI CA180134, The Mary Kay Foundation, UNC University Cancer Research Fund (UCRF); SM: UNC UCRF; JAG: NIH/NIDDK 034987 and DK 056350; LE: Gold Summer Undergraduate Research Fellowship, UNC Honors Research Award; YQ: Sanofi; AC: Royster Society of Fellows, Integrative Vascular Biology Fellowship.

Received: 9 February 2016 Accepted: 22 February 2016

Published online: 19 March 2016

\section{References}

Abounader R, Laterra J (2005) Scatter factor/hepatocyte growth factor in brain tumor growth and angiogenesis. Neuro Oncol 7(4):436-451. doi:10.1215/ s1152851705000050

Baylor Breast Care Center BCoM (2015) CRIZENT: crizotinib and sunitinib in metastatic breast cancer. In: ClinicalTrialsgov [Internet]

Bell LN, Ward JL, Degawa-Yamauchi M, Bovenkerk JE, Jones R, Cacucci BM, Gupta CE, Sheridan C, Sheridan K, Shankar SS, Steinberg HO, March KL, Considine RV (2006) Adipose tissue production of hepatocyte growth factor contributes to elevated serum HGF in obesity. Am J Physiol Endocrinol Metab 291(4):E843-E848. doi:10.1152/ajpendo.00174.2006

Beviglia L, Matsumoto K, Lin C-S, Ziober BL, Kramer RH (1997) Expression of the C-Met/HGF receptor in human breast carcinoma: correlation with tumor progression. Int J Cancer 74(3):301-309. doi:10.1002/ (SICI) 1097-0215(19970620)74:3<301:AID-IJC12>3.0.CO;2-E

Biglia N, Peano E, Sgandurra P, Moggio G, Pecchio S, Maggiorotto F, Sismondi P (2013) Body mass index (BMI) and breast cancer: impact on tumor histopathologic features, cancer subtypes and recurrence rate in pre and postmenopausal women. Gynecol Endocrinol 29(3):263-267. doi:10.3109 /09513590.2012.736559

Biro FM, Deardorff J (2013) Identifying opportunities for cancer prevention during preadolescence and adolescence: puberty as a window of susceptibility. J Adoles Health 52(5 Suppl):S15-S20. doi:10.1016/j. jadohealth.2012.09.019

Boyle P (2012) Triple-negative breast cancer: epidemiological considerations and recommendations. Ann Oncol 23(suppl 6):7-12

Brauer HA, Makowski L, Hoadley KA, Casbas-Hernandez P, Lang LJ, RomanPerez E, D'Arcy M, Freemerman AJ, Perou CM, Troester MA (2013) Impact of tumor microenvironment and epithelial phenotypes on metabolism in breast cancer. Clin Cancer Res 19(3):571-585. doi:10.1158/1078-0432. ccr-12-2123 
Brewster AM, Chavez-MacGregor M, Brown P (2014) Epidemiology, biology, and treatment of triple-negative breast cancer in women of African ancestry. Lancet Oncol 15(13):e625-e634. doi:10.1016/ s1470-2045(14)70364-x

Calle EE, Rodriguez C, Walker-Thurmond K, Thun MJ (2003) Overweight, obesity, and mortality from cancer in a prospectively studied cohort of U.S. adults. N Engl J Med 348(17):1625-1638. doi:10.1056/NEJMoa021423

Camp JT, Elloumi F, Roman-Perez E, Rein J, Stewart DA, Harrell JC, Perou CM, Troester MA (2011) Interactions with fibroblasts are distinct in Basal-like and luminal breast cancers. Mol Cancer Res (MCR) 9(1):3-13. doi:10.1158/1541-7786.MCR-10-0372

Carey LA, Perou CM, Livasy CA, Dressler LG, Cowan D, Conway K, Karaca G, Troester MA, Tse CK, Edmiston S, Deming SL, Geradts J, Cheang MC, Nielsen TO, Moorman PG, Earp HS, Millikan RC (2006) Race, breast cancer subtypes, and survival in the Carolina Breast Cancer Study. JAMA 295(21):2492-2502. doi:10.1001/jama.295.21.2492

Casbas-Hernandez P, Fleming JM, Troester MA (2011) Gene expression analysis of in vitro cocultures to study interactions between breast epithelium and stroma. J Biomed Biotechnol 2011:520987. doi:10.1155/2011/520987

Casbas-Hernandez P, D'Arcy M, Roman-Perez E, Brauer HA, McNaughton K, Miller SM, Chhetri RK, Oldenburg AL, Fleming JM, Amos KD, Makowski L, Troester MA (2013) Role of HGF in epithelial-stromal cell interactions during progression from benign breast disease to ductal carcinoma in situ. Breast Cancer Res (BCR) 15(5):R82. doi:10.1186/bcr3476

Choi WW, Lewis MM, Lawson D, Yin-Goen Q, Birdsong GG, Cotsonis GA, Cohen C, Young AN (2005) Angiogenic and lymphangiogenic microvessel density in breast carcinoma: correlation with clinicopathologic parameters and VEGF-family gene expression. Mod Pathol 18(1):143-152. doi:10.1038/modpathol.3800253

Dawood S, Broglio K, Gonzalez-Angulo AM, Kau SW, Islam R, Hortobagyi GN, Cristofanilli M (2008) Prognostic value of body mass index in locally advanced breast cancer. Clin Cancer Res 14(6):1718-1725. doi:10.1158/1078-0432.ccr-07-1479

Dieras V, Campone M, Yardley DA, Romieu G, Valero V, Isakoff SJ, Koeppen H, Wilson TR, Xiao Y, Shames DS, Mocci S, Chen M, Schmid P (2015) Randomized, phase II, placebo-controlled trial of onartuzumab and/ or bevacizumab in combination with weekly paclitaxel in patients with metastatic triple-negative breast cancer. Ann Oncol 26(9):1904-1910. doi:10.1093/annonc/mdv263

Ding S, Merkulova-Rainon T, Han ZC, Tobelem G (2003) HGF receptor upregulation contributes to the angiogenic phenotype of human endothelial cells and promotes angiogenesis in vitro. Blood 101(12):4816-4822. doi:10.1182/blood-2002-06-1731

Elliott BE, Hung WL, Boag AH, Tuck AB (2002) The role of hepatocyte growth factor (scatter factor) in epithelial mesenchymal transition and breast cancer. Can J Physiol Pharmacol 80(2):91-102. doi:10.1139/y02-010

Ford NA, Devlin KL, Lashinger LM, Hursting SD (2013) Deconvoluting the obesity and breast cancer link: secretome, soil and seed interactions. J Mammary Gland Biol neoplasia 18(3-4):267-275. doi:10.1007/ s10911-013-9301-9

Garner OB, Bush KT, Nigam KB, Yamaguchi Y, Xu D, Esko JD, Nigam SK (2011) Stage-dependent regulation of mammary ductal branching by heparan sulfate and HGF-cMet signaling. Dev Biol 355(2):394-403

Gastaldi S, Comoglio PM, Trusolino L (2010) The Met oncogene and basallike breast cancer: another culprit to watch out for. Breast Cancer Res 12(4):208

Green JE, Shibata M-A, Yoshidome K, M-I Liu, Jorcyk C, Anver MR, Wigginton J, Wiltrout R, Shibata E, Kaczmarczyk S (2000) The C3 (1)/SV40 T-antigen transgenic mouse model of mammary cancer: ductal epithelial cell targeting with multistage progression to carcinoma. Oncogene 19(8):1020-1027

Hiratsuka A, Adachi H, Fujiura Y, Yamagishi S-I, Hirai Y, Enomoto M, Satoh A, Hino A, Furuki K, Imaizumi T (2005) Strong association between serum hepatocyte growth factor and metabolic syndrome. J Clin Endocrinol Metab 90(5):2927-2931. doi:10.1210/jc.2004-1588

lyengar NM, Hudis CA, Dannenberg AJ (2015) Obesity and cancer: local and systemic mechanisms. Annu Rev Med 66:297-309. doi:10.1146/ annurev-med-050913-022228

J-i Yamashita, Ogawa M, S-i Yamashita, Nomura K, Kuramoto M, Saishoji T, Shin S (1994) Immunoreactive hepatocyte growth factor is a strong and independent predictor of recurrence and survival in human breast cancer. Cancer Res 54(7):1630-1633

Jin L, Fuchs A, Schnitt SJ, Yao Y, Joseph A, Lamszus K, Park M, Goldberg ID, Rosen EM (1997) Expression of scatter factor and c-met receptor in benign and malignant breast tissue. Cancer 79(4):749-760. doi:10.1002/ (SICI) 1097-0142(19970215)79:4<749::AID-CNCR12>3.0.CO;2-\#

Johnson AR, Justin Milner J, Makowski L (2012) The inflammation highway: metabolism accelerates inflammatory traffic in obesity. Immunol Rev 249(1):218-238

Lin EY, Pollard JW (2007) Tumor-associated macrophages press the angiogenic switch in breast cancer. Cancer Res 67(11):5064-5066. doi:10.1158/00085472.CAN-07-0912

Liu S (2015) HGF-MET as a breast cancer biomarker. Aging (Albany, NY) 7(3):150-151

Mizuno S, Nakamura T (2013) HGF-MET cascade, a key target for inhibiting cancer metastasis: the impact of NK4 discovery on cancer biology and therapeutics. Int J Mol Sci 14(1):888-919. doi:10.3390/ijms14010888

Olson LK, Tan Y, Zhao Y, Aupperlee MD, Haslam SZ (2010) Pubertal exposure to high fat diet causes mouse strain-dependent alterations in mammary gland development and estrogen responsiveness. Int J Obes (2005) 34(9):1415-1426. doi:10.1038/ijo.2010.51

Puri N, Khramtsov A, Ahmed S, Nallasura V, Hetzel JT, Jagadeeswaran R, Karczmar G, Salgia R (2007) A selective small molecule inhibitor of c-Met, PHA665752, inhibits tumorigenicity and angiogenesis in mouse lung cancer xenografts. Cancer Res 67(8):3529-3534. doi:10.1158/0008-5472. can-06-4416

Sam MR, Elliott BE, Mueller CR (2007) A novel activating role of SRC and STAT3 on HGF transcription in human breast cancer cells. Mol Cancer 6:69. doi:10.1186/1476-4598-6-69

Sameni M, Tovar EA, Essenburg CJ, Chalasani A, Linklater ES, Borgman A, Cherba DM, Anbalagan A, Winn ME, Graveel CR, Sloane BF (2015) Cabozantinib (XL184) inhibits growth and invasion of preclinical TNBC models. Clin Cancer Res. doi:10.1158/1078-0432.ccr-15-0187

Schneider B (2016) Randomized controlled trial of genomically directed therapy in patients with triple negative breast cancer. In: ClinicalTrialsgov [Internet]

Sharma N, Adjei AA (2011) In the clinic: ongoing clinical trials evaluating c-MET-inhibiting drugs. Ther Adv Med Oncol 3(1 Suppl):S37-S50. doi:10.1177/1758834011423403

Shojaei F, Lee JH, Simmons BH, Wong A, Esparza CO, Plumlee PA, Feng J, Stewart AE, Hu-Lowe DD, Christensen JG (2010) HGF/c-Met acts as an alternative angiogenic pathway in sunitinib-resistant tumors. Cancer Res 70(24):10090-10100. doi:10.1158/0008-5472.can-10-0489

Stella MC, Trusolino L, Pennacchietti S, Comoglio PM (2005) Negative feedback regulation of met-dependent invasive growth by Notch. Mol Cell Biol 25(10):3982-3996. doi:10.1128/mcb.25.10.3982-3996.2005

Stewart DA, Yang Y, Makowski L, Troester MA (2012) Basal-like breast cancer cells induce phenotypic and genomic changes in macrophages. Mol Cancer Res 10(6):727-738. doi:10.1158/1541-7786.mcr-11-0604

Sundaram S, Freemerman AJ, Johnson AR, Milner JJ, McNaughton KK, Galanko JA, Bendt KM, Darr DB, Perou CM, Troester MA, Makowski L (2013a) Role of HGF in obesity-associated tumorigenesis: C3(1)-TAg mice as a model for human basal-like breast cancer. Breast Cancer Res Treat 142(3):489-503. doi:10.1007/s10549-013-2741-5

Sundaram S, Johnson AR, Makowski L (2013b) Obesity, metabolism and the microenvironment: Links to cancer. J Carcinog 12:19. doi:10.4103/1477-3163.119606

Sundaram S, Freemerman AJ, Kirk EL, Galanko JA, McNaughton KK, Bendt KM, Darr DB, Troester MA, Makowski L (2014a) Obesity-mediated regulation of HGF/c-Met and reduced basal-like breast cancer latency in parous mice. Cancer Res 74(19 Supplement):4871

Sundaram S, Le TL, Essaid L, Freemerman AJ, Huang MJ, Galanko JA, McNaughton KK, Bendt KM, Darr DB, Troester MA, Makowski L (2014b) Weight loss reversed obesity-induced HGF/c-Met pathway and basal-like breast cancer progression. Front Oncol 4:175. doi:10.3389/ fonc.2014.00175

Swierczynski J, Korczynska J, Goyke E, Adrych K, Raczynska S, Sledzinski Z (2005) Serum hepatocyte growth factor concentration in obese women decreases after vertical banded gastroplasty. Obes Surg 15(6):803-808. doi:10.1381/0960892054222678 
Tolaney SM, Tan S, Guo H, Barry W, Van Allen E, Wagle N, Brock J, Larrabee K, Paweletz C, Ivanova E, Janne P, Overmoyer B, Wright JJ, Shapiro GI, Wine EP, Krop IE (2015) Phase II study of tivantinib (ARQ 197) in patients with metastatic triple-negative breast cancer. Invest New Drugs 33(5):11081114. doi:10.1007/s10637-015-0269-8

Tuck AB, Park M, Sterns EE, Boag A, Elliott BE (1996) Coexpression of hepatocyte growth factor and receptor (Met) in human breast carcinoma. Am J Pathol 148(1):225-232

Wagatsuma S, Konno R, Sato S, Yajima A (1998) Tumor angiogenesis, hepatocyte growth factor, and c-Met expression in endometrial carcinoma. Cancer 82(3):520-530

Wang Y, Selden AC, Morgan N, Stamp GW, Hodgson HJ (1994) Hepatocyte growth factor/scatter factor expression in human mammary epithelium. Am J Pathol 144(4):675-682

WHO (2014) GLOBOCAN: estimated cancer incidence, mortality, and prevalence worldwide in 2012. IARC. http://globocan.iarc.fr/Pages/fact_sheets_ population.aspx
Yan S, Jiao X, Zou H, Li K (2015) Prognostic significance of c-Met in breast cancer: a meta-analysis of 6010 cases. Diagn Pathol 10:62. doi:10.1186/ s13000-015-0296-y

You WK, Sennino B, Williamson CW, Falcon B, Hashizume H, Yao LC, Aftab DT, McDonald DM (2011) VEGF and c-Met blockade amplify angiogenesis inhibition in pancreatic islet cancer. Cancer Res 71(14):4758-4768. doi:10.1158/0008-5472.can-10-2527

Zou HY, Li Q, Lee JH, Arango ME, McDonnell SR, Yamazaki S, Koudriakova TB, Alton G, Cui JJ, Kung PP, Nambu MD, Los G, Bender SL, Mroczkowski B, Christensen JG (2007) An orally available small-molecule inhibitor of c-Met, PF-2341066, exhibits cytoreductive antitumor efficacy through antiproliferative and antiangiogenic mechanisms. Cancer Res 67(9):44084417. doi:10.1158/0008-5472.can-06-4443

\section{Submit your manuscript to a SpringerOpen ${ }^{\odot}$ journal and benefit from:}

- Convenient online submission

- Rigorous peer review

- Immediate publication on acceptance

- Open access: articles freely available online

- High visibility within the field

- Retaining the copyright to your article

Submit your next manuscript at $\boldsymbol{\wedge}$ springeropen.com 